\title{
Evaluation of the effect of glucosamine administration on biomarkers of cartilage and bone metabolism in bicycle racers
}

\author{
REI MOMOMURA ${ }^{1}$, KIYOHITO NAITO $^{1,2}$, MAMORU IGARASHI $^{3}$, TAIJI WATARI ${ }^{1}$, ATSUHIKO TERAKADO ${ }^{4}$ \\ SHINJI OIKE ${ }^{5}$, KOJI SAKAMOTO ${ }^{6}$, ISAO NAGAOKA ${ }^{3}$ and KAZUO KANEKO ${ }^{1}$ \\ ${ }^{1}$ Department of Medicine for Motor Organs, Juntendo University Graduate School of Medicine, Tokyo 113-8421; \\ ${ }^{2}$ Department of Orthopedic Surgery, Juntendo University Shizuoka Hospital, Shizuoka 410-2295; \\ Departments of ${ }^{3}$ Host Defense and Biochemical Research and ${ }^{4}$ Rehabilitation Medicine, \\ Juntendo University Graduate School of Medicine, Tokyo 113-8421; ${ }^{5}$ Japan Keirin School, \\ Shizuoka 410-2402; ${ }^{6}$ Koyo Chemical Co., Ltd., Tokyo 102-0072, Japan
}

Received September 24, 2012; Accepted January 7, 2013

DOI: $10.3892 / \mathrm{mmr} .2013 .1289$

\begin{abstract}
In the present study, the effect of glucosamine administration (1.5 or $3 \mathrm{~g} /$ day) on cartilage and bone metabolism was investigated in bicycle racers, using cartilage- and bone-specific biomarkers, including C-terminal cross-linked telopeptides of type II collagen (CTX-II), C-terminal propeptides of type II procollagen (CPII), N-terminal telopeptides of bone-specific type I collagen (NTx) and bone alkaline phosphatase (BAP). The results indicate that CPII (a marker of type II collagen synthesis) was not substantially changed, however, CTX-II (a marker of type II degradation) was reduced by glucosamine administration, particularly at a dose of $3 \mathrm{~g} / \mathrm{day}$. Consistent with these observations, the ratio of CTX-II/CPII was reduced by glucosamine administration and the effect of glucosamine was dose-dependent. By contrast, the levels of NTx (a bone resorption marker) and BAP (a bone formation marker) were not altered by glucosamine administration. A previous study by this group reported that glucosamine exerts a chondroprotective action in soccer players by preventing type II collagen degradation but maintaining type II collagen synthesis. Together these observations indicate that glucosamine may exert a chondroprotective action by preventing type II collagen degradation in athletes of various sports, including soccer players and bicycle racers.
\end{abstract}

\section{Introduction}

The frequency and severity of joint loading are important determinants for the development of joint destruction

Correspondence to: Professor Isao Nagaoka, Department of Host Defense and Biochemical Research, Juntendo University, Graduate School of Medicine, 2-1-1 Hongo, Bunkyo-ku, Tokyo 113-8421, Japan E-mail: nagaokai@juntendo.ac.jp

Key words: glucosamine, cartilage metabolism, bone metabolism, biomarker, bicycle racer, athlete characterized by degeneration of articular cartilage. The excessive motion and exposure of load on the joints are known to clinically and experimentally cause articular cartilage injury (1-8). Thus, sports associated with repetitive impact and torsional loading on the joints increase the risk of articular cartilage degeneration, which results in the clinical symptoms of osteoarthritis (OA) $(6,7)$. The disease process of OA involves degradation and functional loss of joint cartilage. Previous studies with experimental OA models have revealed that the early changes in the biochemical properties and metabolism of cartilage matrix may be detected prior to the appearance of radiological changes (3). Therefore, a number of biomarkers with greater reliability and sensitivity have been established for the early diagnosis or prognosis of OA (9-16). Furthermore, such biomarkers are used for evaluating the actions of disease-modifying drugs since they specifically reflect alterations in the metabolism of cartilage and associated tissues, including bone and synovium (15). In this context, C-terminal cross-linked telopeptides of type II collagen (CTX-II) and C-terminal propeptides of type II procollagen (CPII) have been used as cartilage-specific type II collagen degradation and synthesis markers, respectively (12-14). In addition, $\mathrm{N}$-terminal telopeptides of bone-specific type I collagen (NTx) and bone alkaline phosphatase (BAP) have been used as markers of bone resorption and formation, respectively.

Among sports of various intensities and frequencies of joint loading, bicycle racing is associated with characteristic biomechanics (i.e., the relatively fixed body position) and low impact on the skeleton, which results in relatively low strain magnitudes and low osteogenic stimulus to the bones. In addition, bicycle racers with low bone mineral density (BMD) have been identified to be at risk of developing osteoporosis at a younger age, as these individuals experience bone loss due to the low mechanical loading nature of the sport. Thus, the effect of bicycle racing on the joints is largely evaluated using BMD and/or bone biomarkers rather than cartilage biomarkers (17-21).

Nutritional supplements, including glucosamine, chondroitin and collagen, are used for joint health to treat or prevent 
sports-related cartilage injuries (i.e., OA) in athletes (22-27). Among these, glucosamine, a naturally occurring amino monosaccharide, has been widely used to treat $\mathrm{OA}$ in humans (28-30). Glucosamine is present in the connective and cartilage tissues as a component of glycosaminoglycans (proteoglycans) and contributes to maintaining the strength, flexibility and elasticity of these tissues. Glucosamine inhibits degradation and stimulates synthesis of glycosaminoglycans, thereby exhibiting chondroprotective actions $(27,31)$. In addition, glucosamine inhibits the expression of collagen-degrading enzymes, matrix metalloproteinases (MMPs), but augments the synthesis of type II collagen in chondrocytes $(32,33)$. Therefore, glucosamine has been hypothesized to exert a chondroprotective action on cartilage injuries by retaining not only proteoglycans, but also type II collagen in the articular cartilage. Previously, we revealed that glucosamine administration reduced enhanced levels of CTX-II but retained CPII levels in soccer players, indicating that glucosamine exerts a chondroprotective action in athletes (soccer players) by preventing type II collagen degeneration but maintaining type II collagen synthesis (34). Based on these observations, we hypothesized that glucosamine administration may affect cartilage metabolism in bicycle racers. In the present study, the effects of glucosamine administration on cartilage and bone metabolism was investigated in bicycle racers, using cartilageand bone-specific biomarkers, including CTX-II, CPII, NTx and BAP.

\section{Materials and methods}

Subjects. A total of 41 competitive bicycle racers belonging to the Japan Keirin School [Shizuoka, Japan; all males, aged between 19 and 22 years old (mean \pm SD, 20.3 \pm 0.9 )] were recruited. Individual heights and weights were measured to determine body mass index (BMI; Table I). The study protocol was approved by the local ethics committee (Juntendo University Shizuoka Hospital, Shizuoka, Japan) and was conducted in accordance with the Declaration of Helsinki and Ethical Guidelines for Epidemiological Research. Subjects provided written informed consent prior to participation in the study. All racers were living in dormitories with similar sleeping patterns and meal times. Subjects were actively training for competitive cycling during the study period over six sessions a week (Monday to Saturday) for $5 \mathrm{~h} /$ day. Participants did not exhibit symptoms of joint injuries, including pain, stiffness and disability and were not consuming medications likely to affect cartilage and bone metabolism during the study period.

Glucosamine administration. To evaluate the effect of glucosamine administration on the biomarkers for cartilage and bone metabolism, individuals were orally administered glucosamine hydrochloride $(500 \mathrm{mg} / \mathrm{capsule}$; supplied by Koyo Chemical Co., Ltd., Tokyo, Japan) at doses of $1.5 \mathrm{~g} /$ day (1.5 g following supper) or $3 \mathrm{~g} /$ day (1.5 $\mathrm{g}$ following breakfast and $1.5 \mathrm{~g}$ following supper) or cornstarch $(0.9 \mathrm{~g} /$ day $)$ as a placebo for 3 months. Groups were classified and matched for age, height, weight and BMI. Serum and urine samples were collected prior to (at month 0 ) and following the glucosamine administration (at month 3).
Measurement of biomarkers. To measure biomarkers of cartilage and bone metabolism, second morning void urine and fasting blood samples were obtained from all subjects. Urine and serum samples were collected following an overnight fast and stored in aliquots at $-80^{\circ} \mathrm{C}$ until use. To evaluate cartilage metabolism, urinary CTX-II and serum CPII were measured. For evaluation of bone metabolism, urinary NTx and serum BAP were analyzed. An assay for CTX-II was performed using a Urine CartiLaps ${ }^{\circledR}$ ELISA kit (Immunodiagnostic System, Inc., Tyne and Wear, UK). CTX-II is cleaved by collagenases during degradation of type II collagen and is used as a type II collagen degradation marker (12). An assay for CPII was performed with a Procollagen type II C-propeptide ELISA kit (Ibex Pharmaceuticals, Inc., Mont-Royal, QC, Canada). CPII is cleaved from type II procollagen during processing of synthesized procollagen and is used as a type II collagen synthesis marker (14). NTx and BAP are used as bone resorption and formation markers, respectively. Urinary NTx, creatinine $(\mathrm{Cr})$ and serum BAP were measured by BML, Inc. (Tokyo, Japan) based on the ELISA (NTx and BAP) and enzyme (Cr) assays. Concentrations of urinary CTX-II and NTx were corrected by $\mathrm{Cr}$ and expressed as $\mathrm{ng} / \mathrm{mmol} \mathrm{Cr}$ and nmol bone collagen equivalent $(\mathrm{BCE}) / \mathrm{mmol} \mathrm{Cr}$, respectively. Concentrations of CPII and BAP were expressed as $\mathrm{ng} / \mathrm{ml}$ and $\mathrm{mg} / \mathrm{l}$, respectively.

Tolerability and safety were assessed throughout the study on the basis of the incidence and severity of intervention-related adverse events (side-effects) as well as abnormal changes in blood pressure, pulse rate and laboratory tests, including hematology and biochemical profiles.

Statistical analysis. Data were analyzed using the SPSS statistical programs (IBM Japan, Ltd., Tokyo, Japan); Friedman test was used for the analysis of the differences between the biomarker levels prior to and following the administration of glucosamine and a two-way analysis of variance (ANOVA) model was used for the analysis of the differences among the three groups (placebo, 1.5 and 3 g glucosamine/day). Data are presented as the mean $\pm \mathrm{SD}$ and $\mathrm{P}<0.05$ was considered to indicate a statistically significant difference.

\section{Results}

In the present study, the effect of glucosamine, a chondroprotective agent for OA, on the levels of biomarkers for type II collagen degradation and synthesis and bone resorption and formation, was examined in bicycle racers. Prior to glucosamine administration, CTX-II levels $(\mathrm{ng} / \mathrm{mmol} \mathrm{Cr}$ ) were $545.2 \pm 331.0,450.6 \pm 93.2$ and $594.4 \pm 369.7$ in placebo $(n=13)$ and $1.5 \mathrm{~g}$ glucosamine/day ( $\mathrm{n}=14)$ and $3 \mathrm{~g}$ glucosamine/day groups ( $\mathrm{n}=14)$, respectively $(\mathrm{P}>0.42)$. CPII levels $(\mathrm{ng} / \mathrm{ml})$ were $1,401.2 \pm 542.9,1,007.1 \pm 329.3$ and $1,113.1 \pm 370.6$ (placebo vs. 1.5 g glucosamine/day, $\mathrm{P}<0.05$; placebo vs. 3 g glucosamine/day, $\mathrm{P}>0.12 ; 1.5 \mathrm{~g}$ glucosamine/day vs. $3 \mathrm{~g}$ glucosamine/ day, $\mathrm{P}>0.79$ ); NTx levels (nmol BCE $/ \mathrm{mmol} \mathrm{Cr}$ ) were 56.2 \pm 23.9 , $65.2 \pm 16.9$ and $63.9 \pm 24.7(\mathrm{P}>0.28)$; BAP levels $(\mathrm{mg} / \mathrm{l})$ were $17.7 \pm 7.4,20.3 \pm 6.2$ and $25.0 \pm 20.9(\mathrm{P}>0.25)$ in placebo and 1.5 and $3 \mathrm{~g}$ glucosamine/day groups, respectively (Table I). Thus, there were no significant differences in the CTX-II, NTx and BAP levels among the placebo, 1.5 and $3 \mathrm{~g}$ glucosamine/ 
Table I. Baseline data of subjects in the placebo and glucosamine groups.

Glucosamine

\begin{tabular}{lcrr} 
Variable & Placebo & $(1.5 \mathrm{~g} /$ day $)$ & $(3 \mathrm{~g} / \mathrm{day})$ \\
\hline Age, years & $22.0 \pm 3.4$ & $21.9 \pm 3.7$ & $21.1 \pm 3.7$ \\
Height, cm & $172.1 \pm 5.4$ & $172.1 \pm 3.3$ & $171.6 \pm 5.1$ \\
Body weight, kg & $73.6 \pm 7.7$ & $73.3 \pm 6.8$ & $73.3 \pm 6.7$ \\
CTX-II, ng/mmol Cr & $545.2 \pm 331.0$ & $450.6 \pm 93.2$ & $594.4 \pm 369.7$ \\
CPII, ng/ml & $1,401.2 \pm 542.9$ & $1,007.1 \pm 329.3$ & $1,113.1 \pm 370.6$ \\
NTX, nmol BCE/mmol Cr & $56.2 \pm 23.9$ & $65.2 \pm 16.9$ & $63.9 \pm 24.7$ \\
BAP, $\mathrm{ng} / \mathrm{ml}$ & $17.7 \pm 7.4$ & $20.3 \pm 6.2$ & $25.0 \pm 20.9$
\end{tabular}

All data are expressed as the mean \pm SD. CTX-II, C-terminal cross-linked telopeptides of type II collagen; CPII, C-terminal propeptides of type II procollagen; NTx, N-terminal telopeptides of bone-specific type I collagen; BAP, bone alkaline phosphatase; Cr, creatinine.

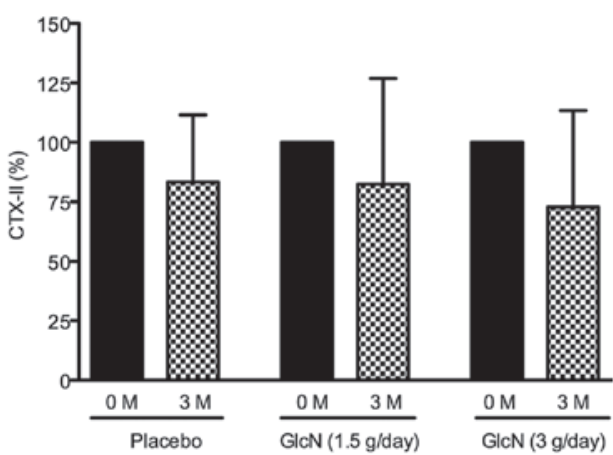

C

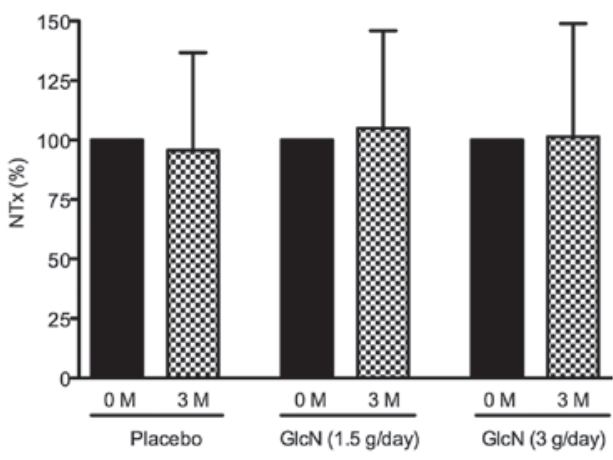

B

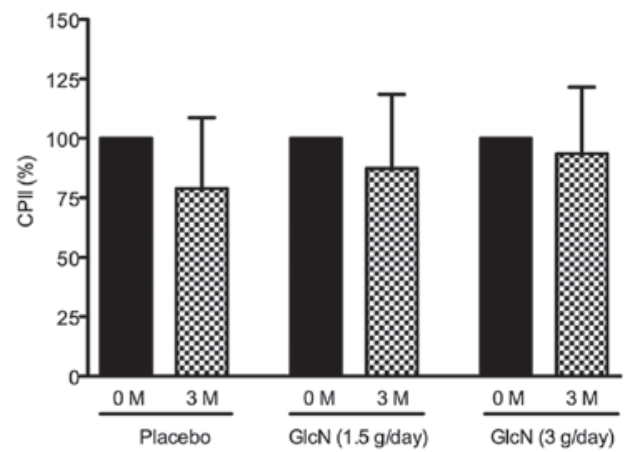

D

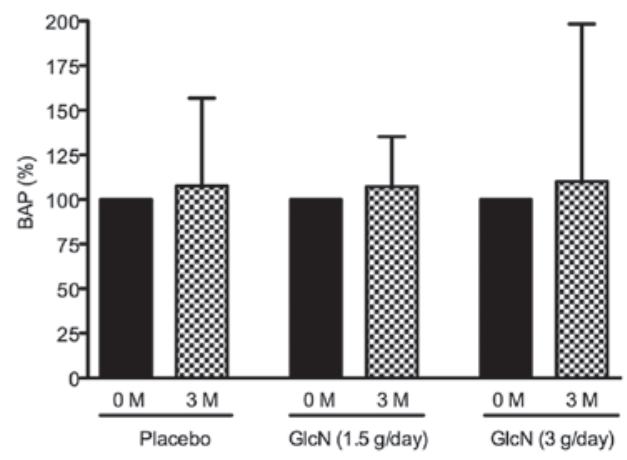

Figure 1. Effect of glucosamine administration on biomarkers of type II collagen degradation and synthesis and bone resorption and formation in bicycle racers. Participants were orally administered placebo or glucosamine ( $\mathrm{GlcN}, 1.5$ or $3 \mathrm{~g} / \mathrm{day})$ for 3 months. Urine and serum samples were collected prior to (0 M) and following (3 M) administration and (A) urinary CTX-II, (B) serum CPII, (C) urinary NTx and (D) serum BAP were measured by ELISA. Levels of urinary CTX-II and NTx were corrected by urinary creatinine. The level of biomarker was expressed as a percentage of that at 0 month. Data are presented as the mean \pm SD. M, month; CTX-II, C-terminal cross-linked telopeptides of type II collagen; CPII, C-terminal propeptides of type II procollagen; NTx, $\mathrm{N}$-terminal telopeptides of bone-specific type I collagen; BAP, bone alkaline phosphatase.

day groups prior to glucosamine administration, although the CPII level in the placebo group was significantly higher than that of the $1.5 \mathrm{~g}$ glucosamine/day group $(\mathrm{P}<0.05)$.

Following the administration of glucosamine, CTX-II levels were decreased by 18 and $23 \%$ from the baseline (prior to administration) in the 1.5 and $3 \mathrm{~g}$ glucosamine/day groups, respectively. CTX-II was also decreased (17\%) in the placebo group after 3 months (Fig. 1A). CPII levels were decreased by 13 and $7 \%$ in the 1.5 and $3 \mathrm{~g}$ glucosamine/day groups, respectively, and was also decreased by $22 \%$ in the placebo group after 3 months (Fig. 1B). By contrast, NTx levels were slightly increased, by 5 and $1 \%$ in the 1.5 and 3 g glucosamine/ day groups, respectively, and decreased by $4 \%$ in the placebo group (Fig. 1C). BAP levels were slightly increased by 8,7 and $10 \%$ in the placebo, 1.5 and 3 g glucosamine/day-groups, respectively (Fig. 1D). 


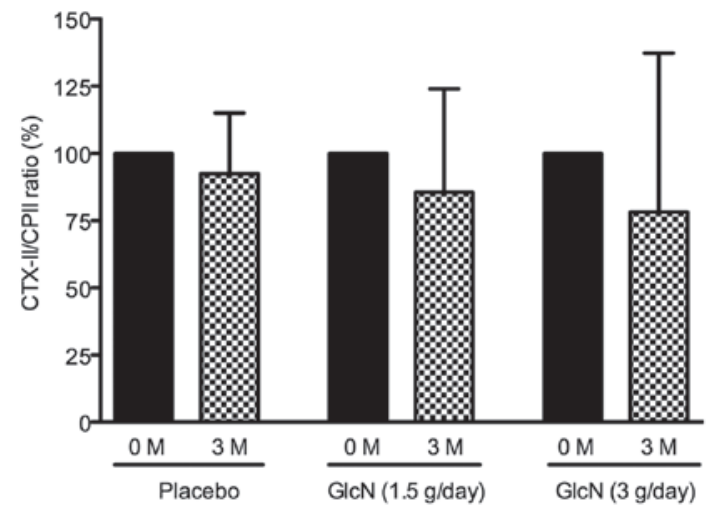

Figure 2. Effect of glucosamine administration on the ratio of type II collagen degradation to synthesis. Bicycle racers were orally administered placebo or glucosamine $(\mathrm{GlcN}, 1.5$ or $3 \mathrm{~g} /$ day) for 3 months and urine and serum samples were collected prior to $(0 \mathrm{M})$ and following $(3 \mathrm{M})$ administration. CTX-II/CPII ratios were calculated using the levels of urinary CTX-II and serum CPII revealed in Fig. 1A and B. The CTX-II/CPII ratio was expressed as a percentage of that at $0 \mathrm{M}$. Data are presented as the mean $\pm \mathrm{SD}$. M, month; CTX-II, C-terminal cross-linked telopeptides of type II collagen; CPII, C-terminal propeptides of type II procollagen.

At present, it is hypothesized that the ratio of type II collagen degradation to synthesis is suitable for the prediction of the progression of joint damage in patients with knee OA $(35,36)$. Based on this concept, the effect of glucosamine administration on the CTX-II/CPII ratio in bicycle racers was evaluated. The CTX-II/CPII ratio was decreased by 14 and $23 \%$ in the 1.5 and $3 \mathrm{~g}$ glucosamine/day groups, respectively but only by $4 \%$ in the placebo group. However, these changes were not identified to be statistically significant (Fig. 2).

In addition, no significant changes were observed in body weight, BMI, blood pressure, pulse rate and results of laboratory tests, including hematological and biochemical profiles (aspartate aminotransferase, alanine aminotransferase, creatinine, blood urea nitrogen, creatine kinase, total cholesterol and glucose) in sera prior to and following glucosamine administration (data not shown).

\section{Discussion}

The majority of athletes $(>70 \%)$ are known to routinely utilize nutritional supplements, including glucosamine, chondroitin, collagen, vitamins, calcium and magnesium (22-27). In the present study, the effect of glucosamine, a chondroprotective agent for OA, on cartilage and bone metabolism was investigated in bicycle racers, using cartilage- and bone-specific biomarkers, including CTX-II, CPII, NTx and BAP.

The mean CTX-II levels were $529.7 \pm 295.8 \mathrm{ng} / \mathrm{mmol} \mathrm{Cr}$ and NTx levels were $66.9 \pm 22.4 \mathrm{nmol} \mathrm{BCE} / \mathrm{mmol} \mathrm{Cr}$ in bicycle racers prior to glucosamine administration $(n=41)$. Previously, we revealed that CTX-II levels $(1,348.0 \pm 212.9 \mathrm{ng} / \mathrm{mmol}$ $\mathrm{Cr}$, mean $\pm \mathrm{SEM})$ and NTx $(92.9 \pm 8.5 \mathrm{nmol} \mathrm{BCE} / \mathrm{mmol} \mathrm{Cr})$ in soccer players $(n=18)$ were significantly higher than the levels of CTX-II $(509.5 \pm 87.6 \mathrm{ng} / \mathrm{mmol} \mathrm{Cr}$, mean \pm SEM) and NTx $(56.0 \pm 8.6 \mathrm{nmol} \mathrm{BCE} / \mathrm{mmol} \mathrm{Cr})$ in non-athlete controls $(n=10)(34)$. Based on these results, CTX-II and NTx levels in bicycle racers are lower compared with soccer players and almost equal to the levels of non-athlete controls, indicating that bicycle racing yields a lower impact on the joints compared with playing soccer.

In addition, we reported that glucosamine may exert a chondroprotective action in soccer players by preventing type II collagen degradation but maintaining type II collagen synthesis (34). The present study also revealed that synthesis of type II collagen (CPII) was not changed by glucosamine administration (13 and 7\% reduction in 1.5 and $3 \mathrm{~g}$ glucosamine and $21 \%$ reduction in placebo groups), however, the degradation of type II collagen (CTX-II) was reduced by glucosamine (18 and $27 \%$ reduction in 1.5 and $3 \mathrm{~g}$ glucosamine/day and $17 \%$ reduction in placebo groups). Consistent with these changes, the ratio of CTX-II (type II collagen degradation)/CPII (type II collagen synthesis) was reduced by glucosamine administration (14 and $22 \%$ reduction in 1.5 and $3 \mathrm{~g}$ glucosamine/day and $8 \%$ reduction in placebo groups). Furthermore, the effect of glucosamine was dose-dependent. These observations indicate that glucosamine may similarly exhibit a chondroprotective action in bicycle racers by preventing type II collagen degradation but maintaining synthesis.

In addition to the chondroprotective action, glucosamine is reported to have the potential to modulate bone metabolism. In this context, we previously identified that glucosamine induces osteoblastic but suppresses osteoclastic cell differentiation in mouse osteoblastic cells and therefore may increase bone matrix deposition and decrease bone resorption, eventually promoting bone formation (37). In addition, glucosamine administration was previously demonstrated to suppress reduction of bone mineral density in a rat experimental OA model (38). The present study indicated that glucosamine administration did not markedly affect NTx (type I collagen degradation) levels but significantly increased BAP (a bone formation marker) levels at $3 \mathrm{~g} /$ day $(\mathrm{P}<0.05)$, when analyzed by Friedman test. These results indicate that glucosamine may have a potential to modulate bone metabolism, particularly bone formation, in humans.

The actions of glucosamine on OA and knee injury in athletes have been largely investigated using a dose of $1.5 \mathrm{~g} /$ day in short- and long-term treatments without apparent adverse effects $(27,30)$. Thus, glucosamine may be a safe and nontoxic agent. Consistent with this, the present study revealed that none of the physical measurement parameters (body weight and body mass index), physiological examinations (systolic/diastolic blood pressure and pulse rate) and laboratory tests (hematology and blood chemistry) were significantly changed, even at a dose of $3 \mathrm{~g} / \mathrm{day}$, following glucosamine administration.

In conclusion, the present study, by the analysis of biomarkers, including CTX-II, CPII, NTx and BAP, demonstrated that cartilage (type II collagen degradation and synthesis) and bone (bone resorption and formation) metabolism in bicycle racers is almost the same as that of non-athletes but lower than that of soccer players. However, the administration of glucosamine reduced type II collagen degradation but maintained synthesis in bicycle racers as well as soccer players. Therefore, glucosamine may exert a chondroprotective action by preventing type II collagen degradation in athletes of various sports, including soccer players and bicycle racers. 


\section{References}

1. O'Kane JW, Hutchinson E, Atley LM and Eyre DR: Sport-related differences in biomarkers of bone resorption and cartilage degradation in endurance athletes. Osteoarthritis Cartilage 14: 71-76, 2006.

2. Roos H, Dahlberg L, Hoerrner LA, Lark MW, Thonar EJ, Shinmei M, Lindqvist U and Lohmander LS: Markers of cartilage matrix metabolism in human joint fluid and serum: the effect of exercise. Osteoarthritis Cartilage 3: 7-14, 1995.

3. Qi C and Changlin H: Effects of moving training on histology and biomarkers levels of articular cartilage. J Surg Res 135: 352-363, 2006

4. Qi C, Changlin $\mathrm{H}$ and Zefeng $\mathrm{H}$ : Matrix metalloproteinases and inhibitor in knee synovial fluid as cartilage biomarkers in rabbits: the effect of high-intensity jumping exercise. J Surg Res 140: 149-157, 2007.

5. Kim HJ, Lee YH and Kim CK: Biomarkers of muscle and cartilage damage and inflammation during a $200 \mathrm{~km}$ run. Eur J Appl Physiol 99: 443-447, 2007.

6. Buckwalter JA and Lane NE: Does participation in sports cause osteoarthritis? Iowa Orthop J 17: 80-89, 1997.

7. Buckwalter JA and Lane NE: Athletics and osteoarthritis. Am J Sports Med 25: 873-881, 1997.

8. Creighton DL, Morgan AL, Boardley D and Brolinson PG: Weight-bearing exercise and markers of bone turnover in female athletes. J Appl Physiol 90: 565-570, 2001.

9. Garnero P, Rousseau JC and Delmas PD: Molecular basis and clinical use of biochemical markers of bone, cartilage and synovium in joint diseases. Arthritis Rheum 43: 953-968, 2000.

10. Garnero P, Piperno M, Gineyts E, Christgau S, Delmas PD and Vignon E: Cross sectional evaluation of biochemical markers of bone, cartilage and synovial tissue metabolism in patients with knee osteoarthritis: relations with disease activity and joint damage. Ann Rheum Dis 60: 619-626, 2001.

11. Poole AR: Biochemical/immunochemical biomarkers of osteoarthritis: utility for prediction of incident or progressive osteoarthritis. Rheum Dis Clin North Am 29: 803-818, 2003

12. Christgau S, Garnero P, Fledelius C, Moniz C, Ensig M, Gineyts E, Rosenquist C and Qvist P: Collagen type II C-telopeptide fragments as an index of cartilage degradation. Bone 29: 209-215, 2001.

13. Poole AR, Ionescu M, Fitzcharles MA and Billinghurst RC: The assessment of cartilage degradation in vivo: development of an immunoassay for the measurement in body fluids of type II collagen cleaved by collagenases. J Immunol Methods 294: 145-153, 2004

14. Shinmei M, Ito K, Matsuyama S, Yoshihara Y and Matsuzawa $K$ Joint fluid carboxy-terminal type II procollagen peptide as a marker of cartilage collagen biosynthesis. Osteoarthritis Cartilage 1: 121-128, 1993.

15. Garnero P and Delmas PD: Biomarkers in osteoarthritis. Curr Opin Rheumatol 15: 641-646, 2003

16. Rousseau JC and Delmas PD: Biological markers in osteoarthritis. Nat Clin Pract Rheumatol 3: 346-356, 2007.

17. Warner SE, Shaw JM and Dalsky GP: Bone mineral density of competitive male mountain and road cyclists. Bone 30: 281-286, 2002 .

18. Smathers AM, Bemben MG and Bemben DA: Bone density comparisons in male competitive road cyclists and untrained controls. Med Sci Sports Exerc 41: 290-296, 2009.

19. Stewart AD and Hannan J: Total and regional bone density in male runners, cyclists and controls. Med Sci Sports Exerc 32: 1373-1377, 2000

20. Nichols JF, Palmer JE and Levy SS: Low bone mineral density in highly trained male master cyclists. Osteoporos Int 14: 644-649, 2003 .
21. Rector RS, Rogers R, Ruebel M and Hinton PS: Participation in road cycling vs running is associated with lower bone mineral density in men. Metabolism 57: 226-232, 2008.

22. Huang SH, Johnson K and Pipe AL: The use of dietary supplements and medications by Canadian athletes at the Atlanta and Sydney Olympic Games. Clin J Sport Med 16: 27-33, 2006.

23. Froiland K, Koszewski W, Hingst J and Kopecky L: Nutritional supplement use among college athletes and their sources of information. Int J Sport Nutr Exerc Metab 14: 104-120, 2004

24. Schwenk TL and Costley CD: When food becomes a drug: nonanabolic nutritional supplement use in athletes. Am J Sports Med 30: 907-916, 2002.

25. Dascombea BJ, Karunaratna M, Cartoon J, Fergie B and Goodmana C: Nutritional supplementation habits and perceptions of elite athletes within a state-based sporting institute. J Sci Med Sport 13: 274-280, 2010.

26. Gorsline RT and Kaeding CC: The use of NSAIDs and nutritional supplements in athletes with osteoarthritis: prevalence, benefits and consequences. Clin Sports Med 24: 71-82, 2005.

27. Ostojic SM, Arsic M, Prodanovic S, Vukovic J and Zlatanovic M: Glucosamine administration in athletes: effects on recovery of acute knee injury. Res Sports Med 15: 113-124, 2007.

28. McAlindon TE,Lavalley MP, Gulin JP and Felson DT: Glucosamine and chondroitin for treatment of osteoarthritis: a systematic quality assessment and meta-analysis. JAMA 283: 1469-1475, 2000.

29. Reginster JY, Deroisy R, Rovati LC, Lee RL, Lejeune E, Bruyere O, Giacovelli G, Henrotin Y, Dacre JE and Gossett C: Long-term effects of glucosamine sulphate on osteoarthritis progression: a randomized, placebo-controlled clinical trial. Lancet 357: 251-256, 2001.

30. Pavelká K, Gatterová J, Olejarová M, Machacek S, Giacovelli G and Rovati LC: Glucosamine sulfate use and delay of progression of knee osteoarthritis: a 3-year, randomized, placebo-controlled, double-blind study. Arch Intern Med 162: 2113-2123, 2002.

31. Igarashi M, Kaga I, Takamori Y, Sakamoto K, Miyazawa K and Nagaoka I: Effects of glucosamine derivatives and uronic acids on the production of glycosaminoglycans by human synovial cells and chondrocytes. Int J Mol Med 27: 821-827, 2011.

32. Nakamura H, Shibakawa A, Tanaka M, Kato T and Nishioka K Effects of glucosamine hydrochloride on the production of prostaglandin E2, nitric oxide and metalloproteases by chondrocytes and synoviocytes in osteoarthritis. Clin Exp Rheumatol 22: 293-299, 2004.

33. Derfoul A, Miyoshi AD, Freeman DE and Tuan RS: Glucosamine promotes chondrogenic phenotype in both chondrocytes and mesenchymal stem cells and inhibits MMP-13 expression and matrix degradation. Osteoarthr Cartil 15: 646-655, 2007.

34. Yoshimura M, Sakamoto K, Tsuruta A, Yamamoto T, Ishida K, Yamaguchi $\mathrm{H}$ and Nagaoka I: Evaluation of the effect of glucosamine administration on biomarkers for cartilage and bone metabolism in soccer players. Int J Mol Med 24: 487-494, 2009.

35. Cahue S, Sharma L, Dunlop D, Ionescu M, Song J, Lobanok T, King L and Poole AR: The ratio of type II collagen breakdown to synthesis and its relationship with the progression of knee osteoarthritis. Osteoarthritis Cartilage 15: 819-823, 2007.

36. Sharif M, Kirwan J, Charni N, Sandell LJ, Whittles C and Garnero P: A 5-yr longitudinal study of type IIA collagen synthesis and total type II collagen degradation in patients with knee osteoarthritis - association with disease progression. Rheumatol 46: 938-943, 2007.

37. Igarashi M, Sakamoto K and Nagaoka I: Effect of glucosamine, a therapeutic agent for osteoarthritis, on osteoblastic cell differentiation. Int J Mol Med 28: 373-379, 2011.

38. Wang SX, Laverty S, Dumitriu M, Plaas A and Grynpas MD: The effects of glucosamine hydrochloride on subchondral bone changes in any animal model of osteoarthritis. Arthritis Rheum 56: 1537-1548, 2007. 